\title{
Significance of Applying Thermal Analysis on Research of Energetic Materials Containing Metal Powders
}

\author{
Liu Lu ${ }^{1,2}$, Zhou Lili ${ }^{1}$, Wang Yicun ${ }^{1}$, Hou Guangyue ${ }^{1}$ and Sun Yalun ${ }^{2 *}$ \\ ${ }^{1}$ Department of Mechatronical Engineering, Shandong Institute for Product Quality Inspection, Shandong, China \\ ${ }^{2}$ Department of Mechatronical Engineering, Beijing Institute of Technology, Beijing, China
}

*Corresponding author: Sun Yalun, Department of Mechatronical Engineering, Beijing Institute of Technology, Beijing, China.
Received Date: May 30, 2020

Published Date: June 11, 2020

\begin{abstract}
Insensitive energetic materials have become one of the important trends in the development of conventional weapons. Metal powder is widely used in composite energetic materials and its advantages have been further confirmed. At present, thermal analysis technology is widely used to study the reaction mechanism of energetic materials including metal powder.
\end{abstract}

Keywords: Energetic materials; Metal powder; Thermal analysis technology

\section{Introduction}

Energetic materials, including propellants, explosives, pyrotechnics and so on, are one of the most important components of the conventional weapon system. They not only determine the safety and reliability of the whole lifecycle of the weapon system, but also determine the operational efficiency of the ammunition system. Insensitive energetic materials have become one of the important trends in the development of conventional weapons [14]. With the continuous intersection, development and integration of materials, chemistry and other disciplines and technologies, the processing technology of energetic materials is changing with each passing day. Through the synthesis of new energetic materials, the surface treatment and modification of single energetic crystal materials, and the use of advanced mixing and composite technology of energetic materials, people can not only improve the ability of energetic materials to unexpected stimulation and inherent environment, but also achieve the purpose of improving its sensitivity, performance and target adaptability in the life cycle. Since 2003, Zhao Fengqi and others [5,6] have introduced the application of nano metal powder in propellant. After several years of research, some of the latest research results have further confirmed the good application prospects of nano metal powders. Its advantages are as follows: improve the combustion efficiency of propellant; increase the burning rate of propellant; catalyze thermal decomposition of propellant components.

The combustion process of energetic materials is a complex multi-stage process based on the chemical reaction of condensed phase and gas phase. All the theoretical models describing combustion are based on the mass and energy (heat) transfer of the thermal decomposition process of energetic materials. The study of thermal decomposition chemistry provides basic kinetic and thermodynamic data for this purpose. Literature [7-10] conducted a deep and beneficial discussion on the thermal decomposition kinetic parameters and kinetic compensation effects of RDX and HMX using DSC, DTA (Differential Thermal Analysis) and TGDTG techniques. Therefore, the study of thermal decomposition chemistry of energetic materials is of great significance to the application of energetic materials. 
As a high energy fuel, metal powder is widely used in explosives. A large amount of heat released by oxidation-reduction reaction has become one of the important ways to improve the damage power and range of ammunition.; The combustion stability and durability of pyrotechnics can be improved by adding nano metal powder into pyrotechnics. Adding metal powder to explosive can improve the heat of detonation and the burning rate of propellant. Therefore, the application of nano metal powder can improve the working ability of explosive and the burning performance of propellant. Metal powder is widely used in composite energetic materials. In 2004, Li Yunong et al. [11-14] found that when metal powder was nano-sized, based on the surface effect, the chemical reaction activity of metal powder can be greatly improved, and many more excellent properties can be obtained. The combustion stability and durability of pyrotechnic powder can be improved by adding nano metal powder into pyrotechnic powder; adding metal powder to the explosive can improve the detonation heat, and the work ability can be greatly improved; the burning rate of propellant can be increased at the same time. Therefore, the application of nano metal powder can improve the function of explosives and improve the combustion performance of gunpowder.

However, for a long time, the formulation design of explosives depends on experience or semi experience, which makes people have no clear understanding of the role and influence of metal powder in the reaction of energetic system. In addition, composite energetic materials often contain more than three components. The interaction mechanism between components is very complicated. The existence of physical processes such as melting, or crystallization makes these interactions more whirling. This interaction is the basis for the study of combustion, detonation and deflagration effects of energetic materials mixed systems. At present, thermal analysis technology is widely used to study the reaction mechanism of energetic materials. From the classical isothermal "thermogravimetry" and decomposition gas transformation technology in 1940s to the technology of isotope labeling and isotope effect, as well as the advanced temperature jump Fourier transform infrared (T-jump-FTIR) technology and high pressure differential scanning calorimetry (PDSC) technology [15-18], the thermal behavior of energetic materials has been characterized, and their thermal decomposition kinetics and mechanism have never been studied Interruption. The study of thermal decomposition is the basis of safety technology, risk, stability, use or storage life assessment of energetic materials in production, use, transportation and storage, and also the indispensable premise of basic theoretical research such as combustion and detonation. Thermal decomposition of energetic materials plays an important role in performance evaluation and basic theoretical research.

\section{Acknowledgement}

None.

\section{Conflict of Interest}

No conflict of interest

\section{References}

1. Duncan W, Eric D, Patrick T (2006) Insensitive munitions (IM): a key aspect of improved munitions safety [J]. Chinese Journal of Energetic Materials 14(5): 323-329.

2. Johansen $\emptyset \mathrm{H}$, Kristiansen JD, Gjersøe R, Alf Berg Terje Halvorsen, et al. (2008) RDX and HMX with reduced sensitivity towards shock initiationRS-RDX and RS-HMX [J]. Propellants, Explosives, Pyrotechnics 33(1): 20-24.

3. Spyckerelle C, Eck G, Sjöberg P, Per Sjöberg Anna-Maria Amnéus (2008) Reduced sensitivity RDX obtained from Bachmann RDX [J]. Propellants, Explosives, Pyrotechnics 33(1): 14-19.

4. Kröber H, Teipel U (2008) Crystallization of insensitive HMX [J]. Propellants, Explosives, Pyrotechnics 33(1): 33-36.

5. Zhao Fengqi, Qin Guangming, Cai Bingyuan (2001) Research status and development direction of nanomaterials applied in explosives [J]. Journal of Thermal Explosives 24(4): 61-65.

6. LI Yu nong, HE Jian Jun, LONG Xiao bing (2004) Development of metallic nanometer particles[J]. Powder Metallurgy Industry 14(1):34- 39.

7. XU Hui xiang, FAN Xue Zhong, LIU Guanli (2003) Progress in the application of nanometer materials to propellants[J]. Chinese Journal of Energetic Materials (Han neng Cai liao) 11(2): 94- 98.

8. Liu Ziru, Liu Yan, Fan Xiping, et al. (2004) Thermal decomposition of RDX and HMX I. characteristic quantity of thermal analysis [J]. Journal of explosives 27(2): 632-66

9. Liu Ziru, Yin Cuimei, Liu Yan, et al. (2004) Thermal decomposition of RDX and HMX II. Kinetic parameters and dynamic compensation effect [J]. Journal of explosives 27(4): 69-71.

10. Liu Ziru, Liu Yan, Fan Xiping, et al. (2006) Thermal decomposition of RDX and HMX III: decomposition mechanism [J]. Journal of explosives 29(4): 14-18.3.

11. Liu Ziru (2008) Thermal analysis of energetic materials [M]. Beijing: National Defense Industry Press.

12. LI Yu nong, HE Jian jun, LONG Xiao bing (2004) Development of metallic nanometer particles[J]. Powder Metallurgy Industry 14(1): 34-39.

13.WANG Xiao Feng, HAO Zhong Zhang (2002) New development of explosive technology[J]. Chinese Journal of Explosives and Propellants 25(4): 35-38.

14. SI Lin Hua (2007) Nano metal fuel [J]. Chinese Journal of Chemical Education 1:11-12.

15. ZHANG JIAN, CAO Xiao guo, HUANG Hui ping (2006) Preparation and use application of metal nano powder [J]. Materia ls Revie, 2 (7): 149-151.

16. Chu Shijin, thermal analysis of explosives [M]. Science Press, pp. 2-8.

17. Esenreich N, Pfeil (1983) A Non 2 linear least 2 squares fit of non 2 iso thermal thermoanalytical curves. Reinvestigation of the kinetics of the autocatalytic decomposition of nit rate cellulose [J]. Thermchim Acta 61: 13.

18. Jutier JJ, Harrison Y, Premont S, et al. (1987) A non-isothermal flourier transform infrared degradation study of no to celluloses derived from wood and cotton[J]. J Applied Polymer Science 33(4): 1359. 